\title{
Searching by Rules: Pigeons' (Columba livia) Landmark-Based Search According to Constant Bearing or Constant Distance
}

\author{
Marcia L. Spetch and Tiana B. Rust \\ University of Alberta
}

\author{
Alan C. Kamil and Juli E. Jones \\ University of Nebraska-Lincoln
}

\begin{abstract}
Pigeons (Columba livia) searched for a goal location defined by a constant relative spatial relationship to 2 landmarks. For one group, landmark-to-goal bearings remained constant while distance varied. For another group, landmark-to-goal distances remained constant while direction varied. Birds were trained with 4 interlandmark distances and then tested with 5 novel interlandmark distances. Overall error magnitude was similar across groups and was larger than previously reported for Clark's nutcrackers (Nucifraga columbiana). During training, error magnitude increased with interlandmark distance for constant-bearing but not constant-distance birds. Both groups searched less accurately along the axis parallel to landmarks than along the perpendicular axis. Error magnitude increased with novel extrapolated interlandmark distances but not with novel interpolated distances. Results suggest modest geometric rule learning by pigeons.
\end{abstract}

Many animals use visual landmarks to remember and return to important locations such as a nest or food source. Often the available visual landmarks are not located right at the goal but instead may be some distance away from the goal. In such cases, the animal must encode not only the characteristics of the landmarks necessary for recognition but also certain aspects of the spatial relationship between goal location and the encoded landmarks to use the landmarks to pilot toward and search for the goal. This landmark-based search has been investigated in numerous animals including insects, rodents, birds, and primates (see Healy, 1998, for reviews).

Although the ability of many animals to encode spatial information from a landmark to a goal is well documented, much remains to be known about which aspects of the spatial relations are typically encoded and how flexible the encoding processes are. Consider a case in which the location of a goal is encoded in terms of two nearby landmarks. The spatial information could be en-

Marcia L. Spetch, Department of Psychology, University of Alberta, Edmonton, Alberta, Canada; Tiana B. Rust, Centre for Health Promotion, University of Alberta; Alan C. Kamil and Juli E. Jones, School of Biological Sciences, University of Nebraska-Lincoln.

Juli E. Jones is now at the Department of Psychology, University of Massachusetts.

Some of these data were presented at the meeting of the Canadian Society for Brain, Behaviour and Cognitive Sciences, Edmonton, Alberta, Canada, June 1999, and formed the honors thesis of Tiana B. Rust. This research was supported by Natural Sciences and Engineering Research Council of Canada (NSERC) Research Grant RGPIN 38861 to Marcia L. Spetch, National Institute of Mental Health Grant MH61810 to Alan C. Kamil, and an NSERC summer scholarship to Tiana B. Rust. We thank Jeff Beitel, Debbie Kelly, Lisa Lis, Kate Manchak, Karen Steinbring, and Martha Schultz for assistance with the research.

Correspondence concerning this article should be addressed to Marcia L. Spetch, Department of Psychology, University of Alberta, Edmonton, Alberta T6G 2E9, Canada. E-mail: mspetch@ualberta.ca coded in one of several ways. For example, the two landmarks could be encoded as an array, and the relative geometric location of the goal to the array could be encoded. Alternatively, the animal could encode the distance and direction to the goal independently for one or both of the landmarks. Moreover, this distance and direction information could be encoded as unitary vectors or as independent pieces of information.

In many experimental studies of landmark use, the distance and direction of a landmark or landmark array to the goal are held constant during training and then some transformation of the spatial information is conducted on unreinforced test trials to assess what or how spatial information had been encoded (see Cheng \& Spetch, 1998). These types of transformation tests have suggested that landmark-based search by pigeons is typically characterized by an elemental process in which different pieces of spatial information are independently encoded. For example, Cheng (1994) provided evidence that pigeons encode the directional information provided by a landmark independently from the distance information rather than combining the information into a vector. When multiple landmarks are available, the information from each landmark again appears to be encoded independently even though it may be averaged to determine search location (Cheng, 1995). Finally, when a goal location is specified by an array of landmarks, pigeons appear to learn the location of the goal relative to individual landmarks rather than learning the relative location of the goal in the entire array (Spetch, Cheng, \& MacDonald, 1996; Spetch et al., 1997). For example, Spetch and colleagues $(1996,1997)$ trained pigeons, in both touch-screen and open-field tasks, to find a goal that was in the center of a square array of four identical landmarks. When the landmarks were spread apart on a transformation test, the pigeons did not search in the middle of the expanded array but instead searched in locations that maintained the approximate training distances and directions from individual landmarks. A similar tendency to search on the basis of individual landmarks rather than using the spatial rela- 
tionship of the goal to the whole array was found for gerbils (Collett, Cartwright, \& Smith, 1986) and monkeys (Sutton, Olthof, \& Roberts, 2000), whereas adult humans show the opposite tendency (Spetch et al., 1996, 1997).

Transformation tests like those described above are useful for revealing an animal's typical or preferred encoding strategy, but they do not indicate how flexible that strategy is. One way to determine whether an animal is capable of learning a nonpreferred strategy is to train the animal on a task that can be solved only by using the nonpreferred strategy. For example, training with trialunique stimuli in a matching-to-sample task revealed that pigeons are capable of learning a generalized matching rule even though they are typically more prone to learning specific stimulusresponse rules (Wright, Cook, Rivera, Sands, \& Delius, 1988).

Recently, studies by Kamil and Jones (1997, 2000) have revealed that Clark's nutcrackers are able to learn a geometric rule that specifies the location of a goal in a landmark array. The nutcrackers were trained to find food that was hidden at a fixed location relative to two landmarks that varied across trials in their interlandmark distance. The food was located at the midpoint between the landmarks or at a fixed distance or direction away from the landmarks. The variation in interlandmark distance meant that these tasks could not be solved by learning a single independent rule about the absolute spatial relationship between each landmark and the goal. Instead, accurate search would require that the bird learn either (a) multiple absolute spatial relationships, one for each interlandmark distance experienced during training, or (b) a single geometric rule specifying the relative spatial relationship of the goal to both landmarks. Kamil and Jones found that in most cases the nutcrackers learned the tasks using geometric rules, as evidenced by their ability to search at the appropriate relative locations on transfer tests with new interlandmark distances. The one exception to excellent performance on transfer tests was that nutcrackers trained with a constant-distance rule did not search as accurately at novel interlandmark distances that were extrapolated beyond the training range.

The present study examined whether pigeons are also capable of geometric rule learning and, if so, how similar their performance is to that of nutcrackers. Jones, Anotoniadis, Shettleworth, and Kamil (2002) recently reported that pigeons were capable of learning to search midpoint between two landmarks that varied in interlandmark distance. In the present study, we investigated pigeons' ability to solve a task that involved a more complex relative rule. The goal was centered between but displaced away from the two landmarks such that it formed the third point of a triangle. The interlandmark distance varied from $36 \mathrm{~cm}$ to $108 \mathrm{~cm}$ during training, and novel interlandmark distances ranging from $24 \mathrm{~cm}$ to $120 \mathrm{~cm}$ were presented in testing. For one group (constantbearing group), the goal was always located $45^{\circ}$ northwest of the south landmark and $45^{\circ}$ southwest of the north landmark. To locate the goal, the birds in this group had to vary their distance from the landmarks to maintain the correct direction. For the other group (constant-distance group), the goal was always located $61 \mathrm{~cm}$ away from each landmark on the west side. To locate the goal in this condition, the birds had to vary their direction of search from each landmark to maintain the correct distance.
Method

\section{Subjects}

Eight adult silver king pigeons (Columba livia) served as subjects. The birds were maintained at $85 \%$ of their free-feeding weight throughout the experiment by pigeon pellets obtained after the experimental sessions and by maple peas used as reinforcement during the experimental sessions. The birds were housed in large individual cages $(46 \mathrm{~cm}$ wide, $32 \mathrm{~cm}$ high, and $40 \mathrm{~cm}$ deep) under a 12-hr light-dark cycle with light onset at 6:00 a.m. The birds had free access to water and grit in their home cages.

\section{Apparatus}

The open-field laboratory room measured $300 \mathrm{~cm} \times 330 \mathrm{~cm}$. The walls were white but contained several distinct features: a door and a light switch on the north wall, a window covered with black paper on the east wall, a larger window covered with black paper on the south wall, and a window with one-way glass on the west wall. A wooden tray, which served as the search space, was centered on the south end of the room. The tray measured $2 \mathrm{~m} \times 2 \mathrm{~m}$ with sides $5 \mathrm{~cm}$ high and was filled with sawdust approximately $3 \mathrm{~cm}$ deep. Two pieces of polyvinyl chloride pipe $2.5 \mathrm{~cm}$ in diameter and $40 \mathrm{~cm}$ high served as landmarks. They were always aligned north-south of each other in the search space. The south landmark was painted blue, and the north landmark was painted red. The goal consisted of four maple peas in a small ( $4 \mathrm{~cm}$ diameter) rubber cup. A start-finish box was located on the north side of the tray. A ceiling-mounted video camera was centered above the tray and was connected to a TV and VCR located in an adjacent room. A one-way window in this adjacent room also allowed the experimenter to directly observe the birds while they searched and to manually open and close a door on the start-finish box via a pulley system.

The interlandmark distances used for training were 36, 60, 84, and 108 $\mathrm{cm}$. For the bearing group, the goal was always located at a $45^{\circ}$ angle southwest of the north landmark (red) and $45^{\circ}$ northwest of the south landmark (blue). For the distance group, the goal was always located $61 \mathrm{~cm}$ from each of the landmarks on the west side. Figure 1 shows examples of the landmark-goal relationships in the two conditions. The distance between landmarks and the absolute east-west and north-south locations of the landmarks in the tray varied randomly across trials.

\section{Procedure}

Home-cage training. Prior to placement in the experimental room, the birds were trained in the home cage to find maple peas hidden under sawdust. First, the rubber food cup used in the experiment was placed within the bird's larger food cup (11 cm diameter) in its home cage, and several maple peas were placed inside the rubber food cup. Over trials, the maple peas were partially and then fully covered with sawdust until the bird readily swept away the sawdust to find and eat the peas.

Preliminary training. The bird was placed within the start box at the beginning of each trial. To begin the trial, the experimenter opened the door to the start box from the adjacent room using the pulley system. The bird was allowed to search for the maple peas in the rubber food cup, which was initially placed just outside the start box. After the bird consumed the peas, the door to the finish box (which was baited with maple peas) was raised and remained open until the bird entered. This procedure was repeated over trials, and the food cup was gradually moved to various locations within the search tray. The food cup was then partially covered with sawdust, and this preliminary training continued until the bird readily approached and ate from the partially covered cup regardless of its location and readily entered the finish box when the door was opened.

Training. Four randomly selected birds were assigned to the bearing group, and the remaining 4 were assigned to the distance group. All birds received the same training except for the spatial relationship between the 
A

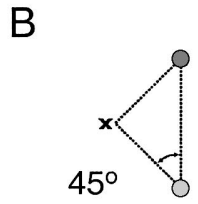

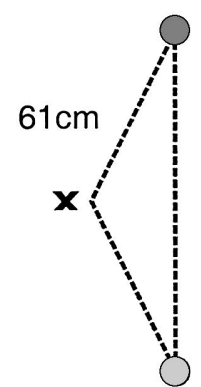

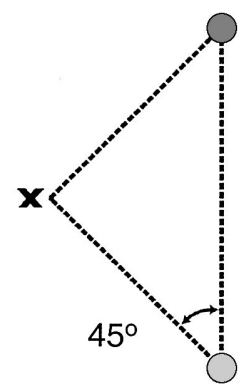

Figure 1. The landmark-goal relationships for the constant-distance (A) and constant-bearing (B) groups. For the distance group, the goal was always located $61 \mathrm{~cm}$ from each of the landmarks on the west side. For the bearing group, the goal was always located $45^{\circ}$ southwest of the north landmark and $45^{\circ}$ northwest of the south landmark.

landmarks and the goal. Trials began with the opening of the start-box door, and the bird was then allowed to search for the goal. If the goal was not located within $10 \mathrm{~min}$, the door of the baited finish box was opened to end the trial. On trials in which the food and food cup were absent (no-goal trials), the baited finish-box door was opened after 10 sweeping movements were recorded or after a maximum of $10 \mathrm{~min}$. Sweeping movements rather than pecking were used as the index of searching because previous research (e.g., Spetch et al., 1997) indicated that pigeons typically sweep away the bedding first and then peck only upon exposing the food. The sweeping movements were operationally defined as a sideways movement of the beak that resulted in visible displacement of bedding material.

In all stages of training, the birds received either one or two sessions, consisting of four trials, per day. The second session, when given, occurred at least $2 \mathrm{hr}$ after the first session. Some birds appeared satiated within a single session; so, a second session was conducted only if the bird found the food or made 10 sweeping movements on all trials of the first session.

In the first stage of training, the cup containing the maple peas was only partially covered with sawdust, and one of the peas was placed on top of the sawdust covering the cup on the first three trials of each session. The goal was then completely buried on the fourth trial. When the birds were able to locate the goal within $4 \mathrm{~min}$ on all trials for three consecutive sessions, they began the second stage of training.

In the second stage of training, the goal was partially covered with sawdust on the first two trials of each session and completely covered on the last two trials of each session. When the birds were able to locate the goal within $4 \mathrm{~min}$ on all trials for three sessions, or after they had completed 70 sessions of Stage 2 training, they began the third stage of training-135 sessions in which the goal was completely buried on all trials.

During the second and third stages of training, the birds also received one no-goal trial each session. During Stage 2, no-goal trials occurred on

either the third or fourth trial of each session; during Stage 3, they occurred on the second, third, or fourth trial. On no-goal trials, both the food and the food dish were absent. The birds were allowed to make 10 sweeping movements on these no-goal trials before the finish box was opened.

Transfer tests. Following training, each bird was given 27 no-goal test trials to examine transfer to novel interlandmark distances. One test trial was presented per session, randomly selected from Trials 2,3 , and 4 . The four training interlandmark distances $(36,60,84$, and $108 \mathrm{~cm})$ and five novel interlandmark distances $(24,48,72,96$, and $120 \mathrm{~cm})$ were presented on 3 test trials each. Three of these novel distances are interpolated between the training distances, and two are extrapolated beyond the training range.

Data recording and reduction. All no-goal trials in Stage 3 were videotaped. The tapes were played back on a television screen. The locations of the landmarks and the sweep responses were marked on transparencies that were overlaid on the screen. To facilitate comparison of the pigeons' performance with that of nutcrackers (Nucifraga columbiana), we scored the data and presented them in a similar fashion to that used by Kamil and Jones (2000). Accuracy was assessed by determining how far away each response was to the location at which the goal would have been had it been present. Separate error distances in the north-south and the east-west axes were measured for each of the sweeps. The north-south axis error is the distance from each sweep location to the imaginary line through the correct location, parallel to the landmarks. The east-west axis error is the distance from each sweep to the imaginary line through the correct location, perpendicular to the line connecting the two landmarks. Total error distance (straight line distance from the search location to the target location) was also measured for each response.
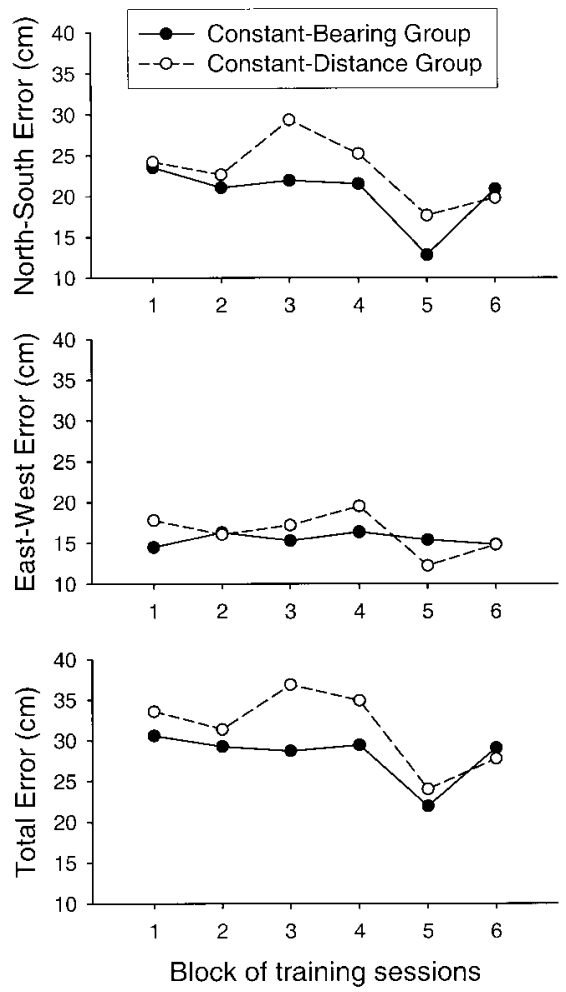

Figure 2. Mean error in the north-south and east-west axes and mean total error for each group as a function of block of training sessions. 

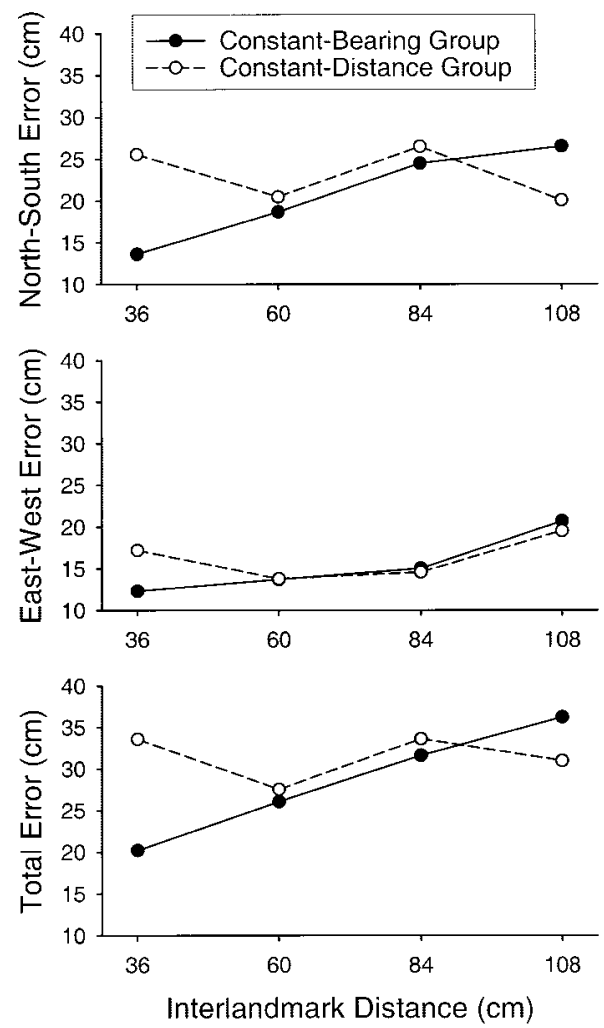

Figure 3. Mean error in the north-south and east-west axes and mean total error for each group as a function of interlandmark distance during training.

Results

\section{Training}

To analyze accuracy during training, we divided the Stage 3 training period into six blocks of 22-23 trials, and then we scored 1 randomly selected no-goal trial for each interlandmark distance within each block. For each trial, error distances were measured separately for each response and then averaged. Figure 2 shows error distances as a function of training block. The data are shown for each group and each axis but are collapsed over interlandmark distance. Error distance was much larger in the north-south axis (parallel to the landmark array) than in the east-west axis, and error distances showed a nonsystematic decrease over training blocks. A mixed-model analysis of variance (ANOVA) on the data shown in the top two panels of Figure 2, with group as a betweensubjects factor and axis and block as within-subject factors, revealed significant main effects of axis, $F(1,6)=53.88, p<.01$, and block, $F(5,30)=3.46, p<.05$. Neither the main effect of group nor any of the interaction terms was significant $(p s>.20)$.

Figure 3 shows error distances for each group and each axis as a function of interlandmark distance collapsed over blocks. A systematic increase in error rate as a function of interlandmark distance is apparent for the bearing group but not for the distance group. A mixed-model ANOVA with group as a between-subjects factor and axis and interlandmark distance as within-subject factors confirmed a significant Group $\times$ Interlandmark Distance interaction, $F(3,18)=3.30, p<.05$. The only other significant effect revealed by this analysis was the main effect of axis, $F(1$, 6) $=103.94, p<.01$.

\section{Transfer Tests}

Approximately halfway through transfer testing, 1 bird in the distance group began to search at the edge of the search tray on all trials, resulting in a failure to consistently find the food within the time limit on baseline trials and very poor accuracy on test trials The bird was placed back on baseline trials only for several sessions but failed to improve and therefore was dropped from the study. None of the transfer data for this bird are included in the figures or analyses.

Figure 4 shows error distance for each group and each axis as a function of interlandmark distance during transfer tests. The interlandmark distances of $24,48,72,96$, and $120 \mathrm{~cm}$ were novel, whereas the remaining distances had been experienced during training. Error distances were highly variable but tended to be slightly larger at the longer interlandmark distances. As in training, the birds showed larger error distances in the north-south axis than in the east-west axis. A mixed-model ANOVA with group, interlandmark distance, and axis as factors revealed significant main effects of interlandmark distance, $F(8,40)=6.74, p<.01$, and axis, $F(1,5)=37.96, p<.01$. No other effects were significant.
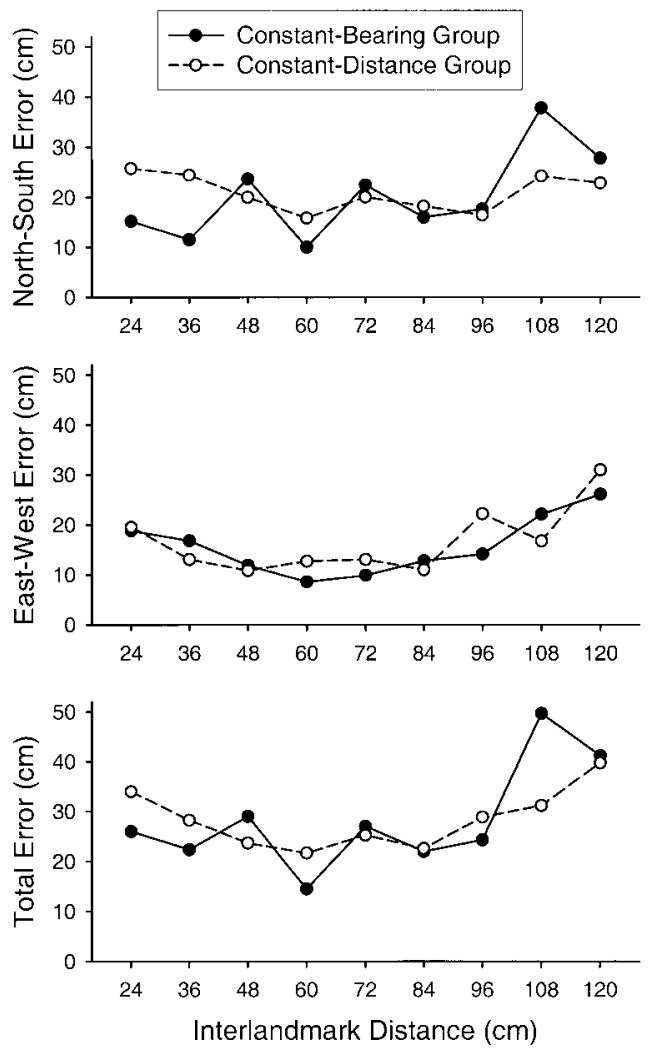

Figure 4. Mean error in the north-south and east-west axes and mean total error for each group as a function of interlandmark distance during transfer testing. 


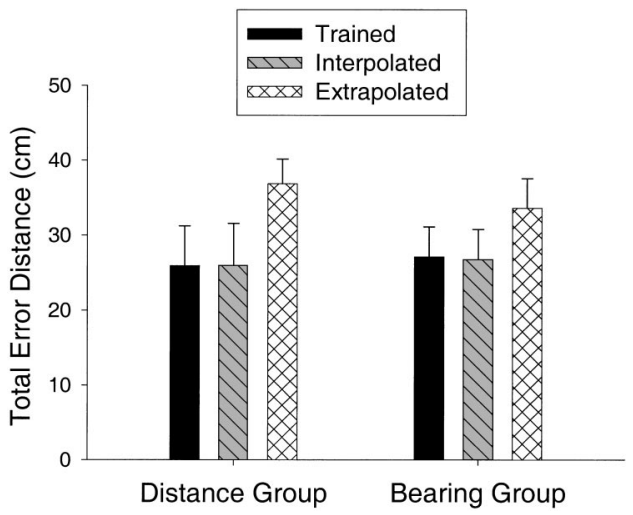

Figure 5. Mean total error for each group averaged across the trained, interpolated, and extrapolated interlandmark distances during transfer testing. Error bars represent the standard errors of the means.

In general, the birds transferred well to novel interlandmark distances that were interpolated between the distances experienced in training but showed increased error for novel interlandmark distances that were extrapolated beyond the training range $(24 \mathrm{~cm}$ and $120 \mathrm{~cm}$ ). Figure 5 shows mean total error scores averaged across the four trained distances, the three novel interpolated distances, and the two novel extrapolated distances for each group. For both groups, error increased only for the extrapolated novel interlandmark distances. A mixed-model ANOVA with group and condition (trained, interpolated, or extrapolated distances) as fac- tors revealed only a significant main effect of condition, $F(2$, $10)=9.72, p<.01$. A post hoc contrast on the means showed a significant difference in error distance between trained and extrapolated distances, $F(2,5)=21.06, p<.01$, but not between trained and interpolated distances, $F(2,5)=0.01, p>.10$.

To provide a more detailed look at the search behavior in each group on transfer tests, we plotted search distributions in the north-south and east-west axes for test trials with trained, interpolated, or extrapolated interlandmark distances (see Figure 6). Error distances along each axis for each of the first five search pecks from each trial for each bird were recorded. The distributions of these errors were then divided into bins (shown along the $x$-axis) with error $(\mathrm{cm})$ divided by interlandmark distance $(\mathrm{cm})$ so that all errors are on a common axis. Thus, the middle bins represent pecks in the hypothetical goal location, and bins outside of the center represent errors of increasing relative magnitude Search behavior for the trained and interpolated interlandmark distances were nicely centered with a peak in the goal region. Search distributions for the extrapolated interlandmark distances showed less accurate or less defined peaks. A comparison between our Figure 6 and Figure 6 in Kamil and Jones's (2000) article also reveals that the frequency of errors far from the goal was considerably higher for the pigeons than for the nutcrackers, particularly in the constant bearing condition.

For a visual depiction of the pigeons' search behavior on training and transfer probe trials, we plotted maps showing the mean location of sweeps during each test for each bird. These maps are shown in Figures 7, 8, and 9.

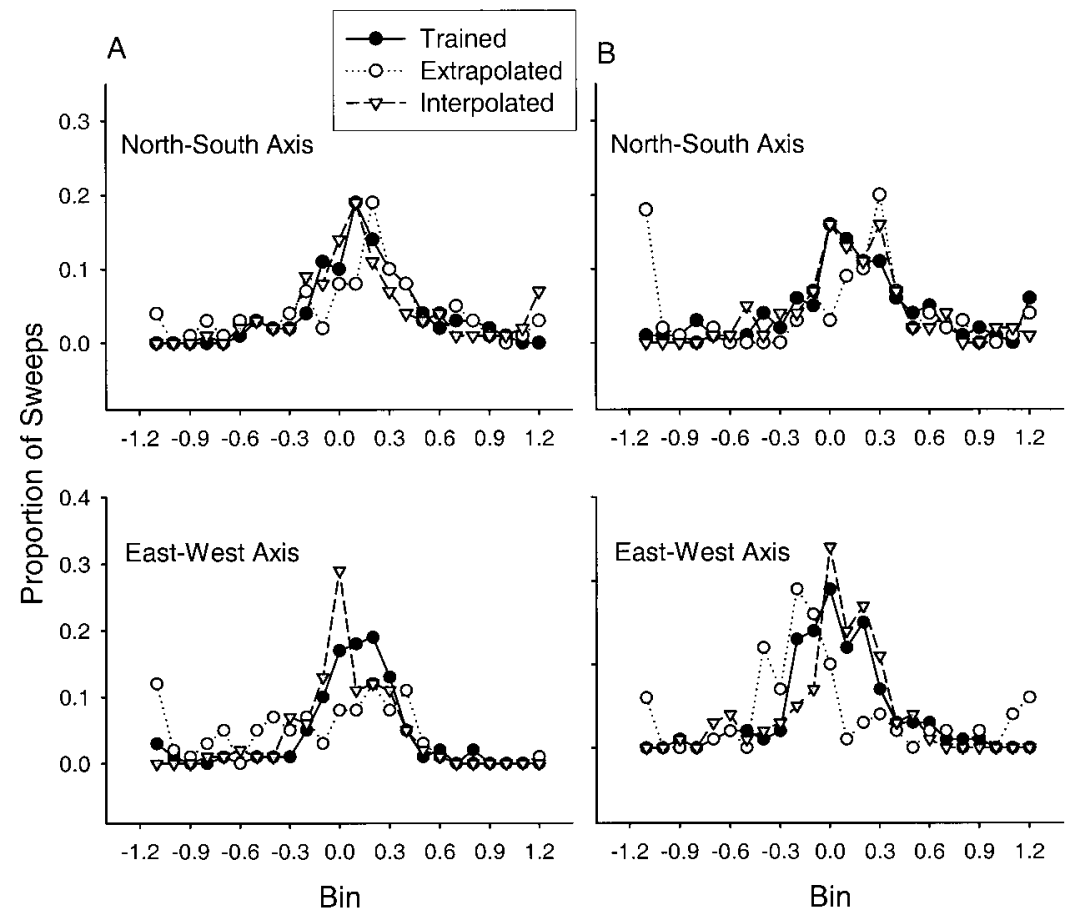

Figure 6. Search distributions in the north-south and east-west axes for the constant-bearing (A) and constant-distance (B) groups during transfer tests. Each of the first five search sweeps from each trial is included. The distributions were divided into bins (shown along the $x$-axis), with error divided by interlandmark distance $(\mathrm{cm})$ so that all errors are on a common axis. 
A
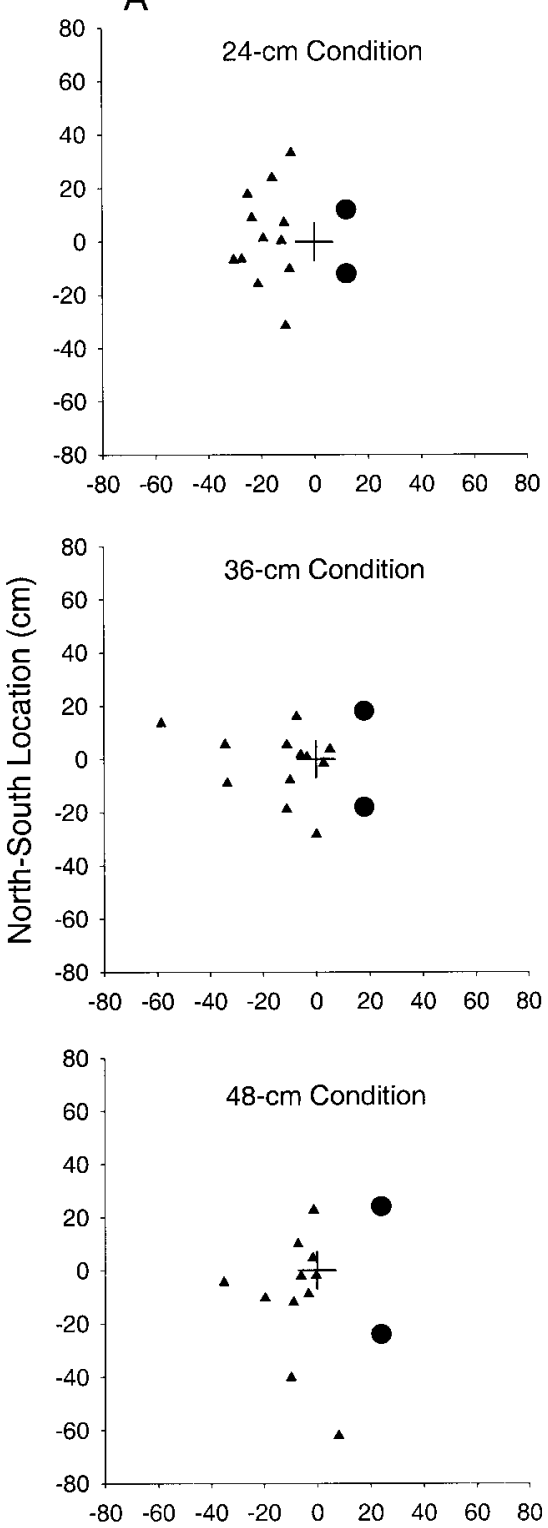

B
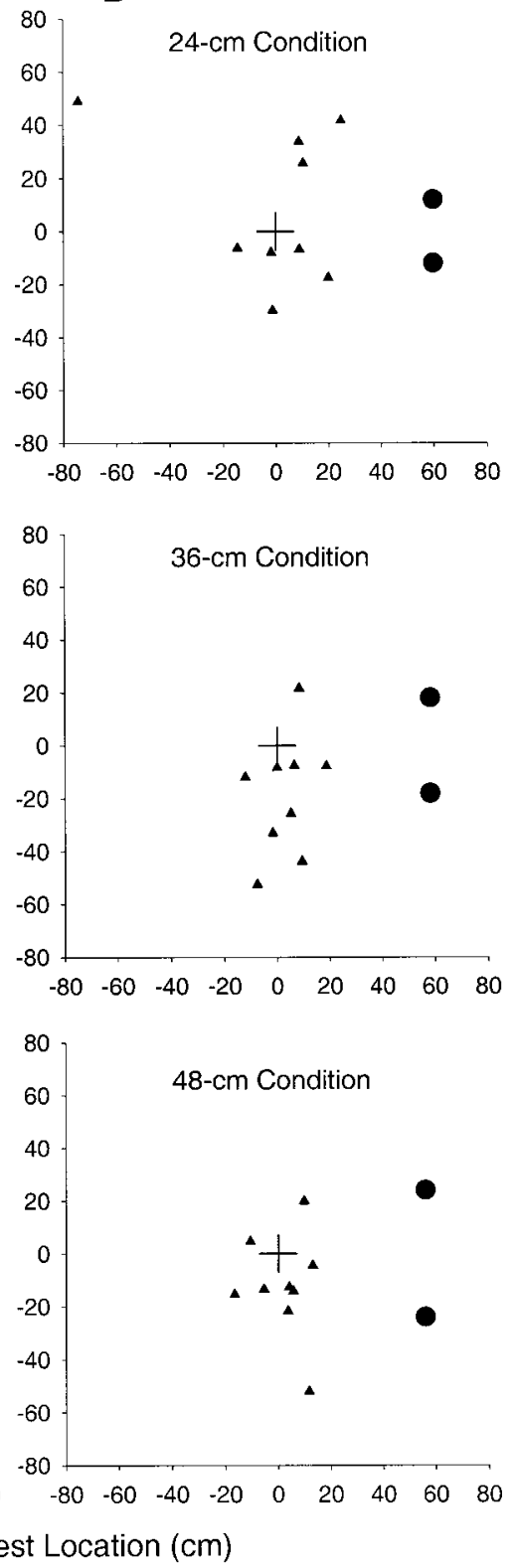

Figure 7. Maps showing the location of searching for each bird in the bearing group (A) and the distance group (B) during probe tests with the 24-cm (extrapolated), 36- $\mathrm{cm}$ (trained), and 48-cm (interpolated) interlandmark distances. Each small triangle represents the mean location of the first five sweeps of a single trial. The large circles show the locations of the landmarks, and the + shows the target location.

\section{Discussion}

These results demonstrate that pigeons can learn to find a goal when the goal location is defined by either a constant-bearing or constant-distance relationship to landmarks and that pigeons can generalize to novel landmark arrays that fall within the trained range. Solving these tasks required the pigeons to vary their distance (constant-bearing group) or their bearing (constantdistance group) from the landmarks across trials. These findings complement other studies showing that spatial encoding in pigeons is flexible (Keeton, 1974; Kelly, Spetch, \& Heth, 1998; Spetch \& Edwards, 1988).

Although pigeons acquired the task, their search behavior appeared to be considerably less accurate and more variable than that found in previous studies in which the absolute distance and bearing from the goal to the landmarks was fixed during training (e.g., Cheng, 1989; Spetch et al., 1997). Numerous procedural differences between this study and previous ones, including a much larger average landmark-to-goal distance, could account for 
A

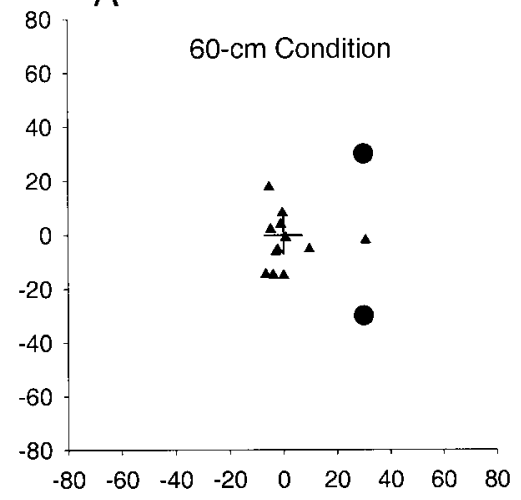

$B$

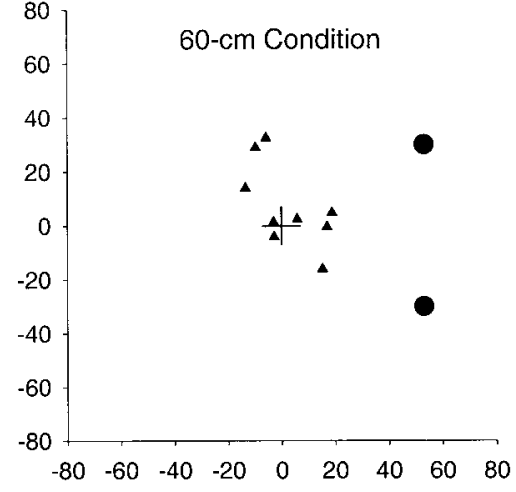

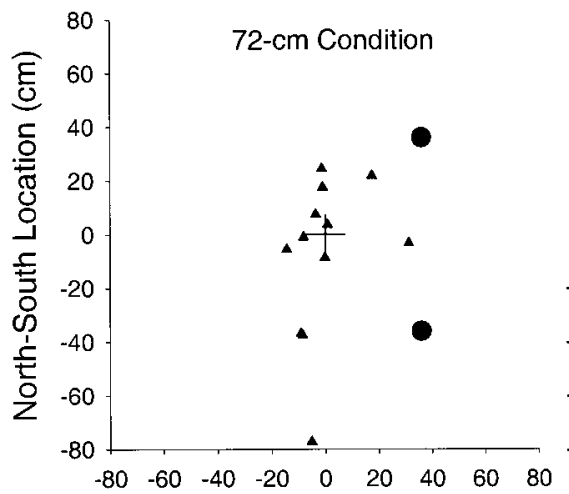
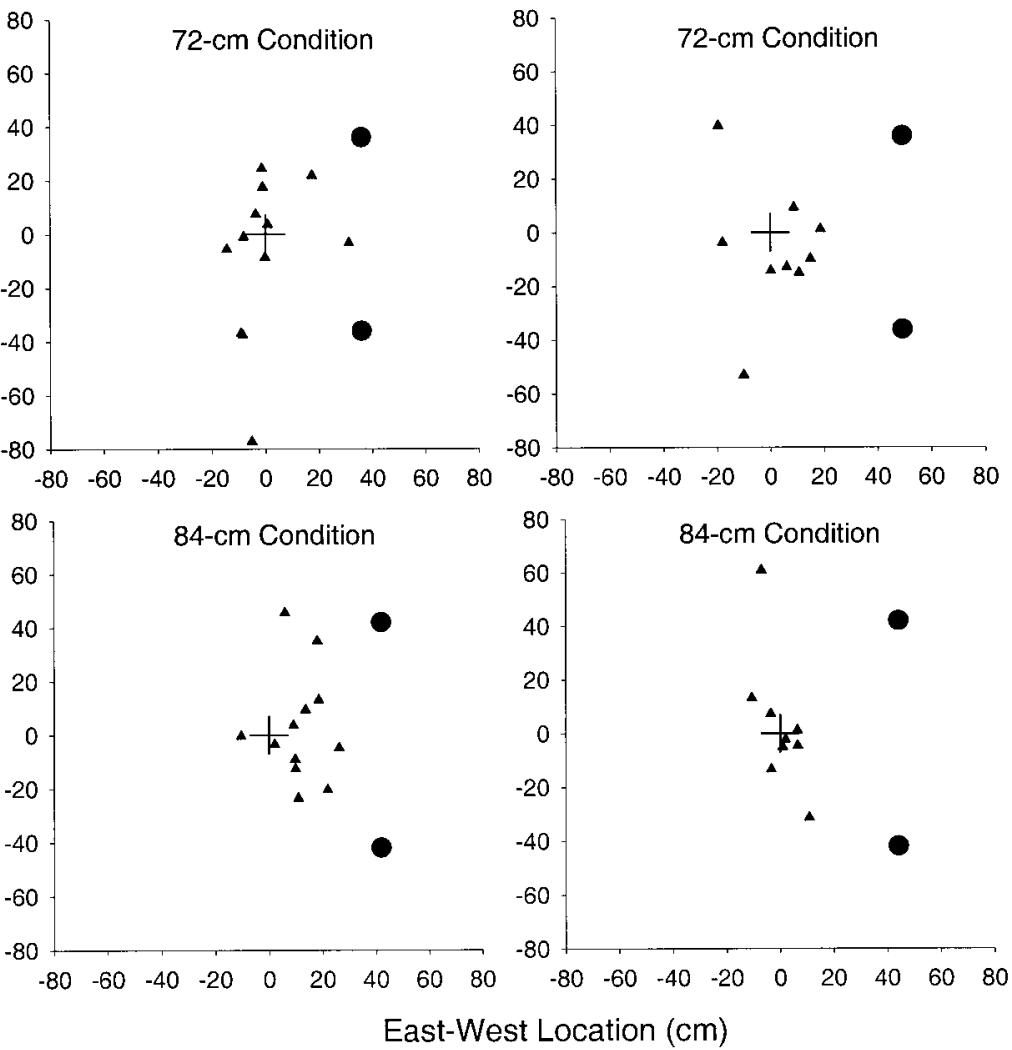

Figure 8. Maps showing the location of searching for each bird in the bearing group (A) and the distance group (B) during probe tests with the 60-cm (trained), 72-cm (interpolated), and 84-cm (trained) interlandmark distances. Each small triangle represents the mean location of the first five sweeps of a single trial. The large circles show the locations of the landmarks, and the + shows the target location.

the lower accuracy in the present study. However, it may be the case that pigeons find relative rules based on variable spatial relationships considerably more difficult to acquire than rules based on absolute spatial relationships.

During training, accuracy decreased with interlandmark distance for the bearing group but not for the distance group, presumably because landmark-to-goal distance increased with interlandmark distance only for the bearing group. There are two possible reasons for this effect, which are complementary rather than mutually exclusive. First, nearer landmarks often exert greater control over search than landmarks that are farther from a goal (e.g., Bennett, 1993; Cheng, 1989; Spetch \& Wilkie, 1994). Second, the accuracy of search when landmarks are close to a goal is likely to be better because of the psychophysics and geometry of the navigational problem (Kamil \& Cheng, 2001). During testing, the relationship between accuracy and interlandmark distance was less apparent for the bearing group, possibly suggesting an effect of experience on overcoming the distance problem. It is interesting to note that, other than the effect of interlandmark distance, no apparent differences between the constant-distance and constant-bearing 
A
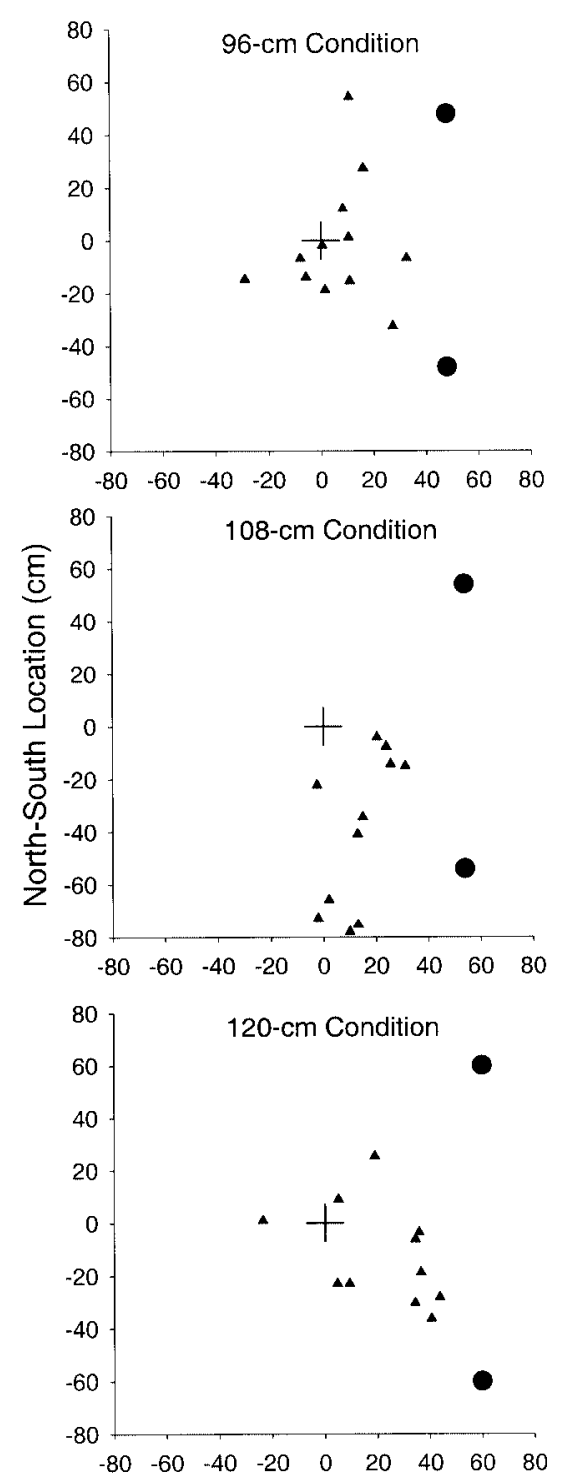

B
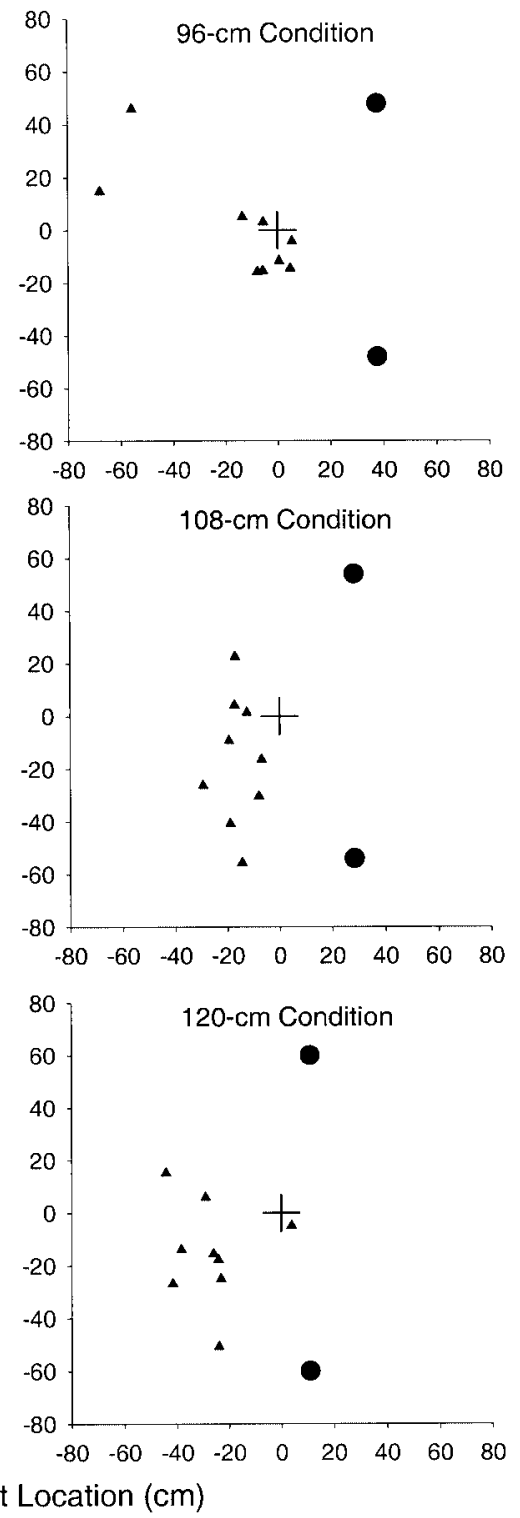

Figure 9. Maps showing the location of searching for each bird in the bearing group (A) and the distance group (B) during probe tests with the $96-\mathrm{cm}$ (interpolated), 108-cm (trained), and 120-cm (extrapolated) interlandmark distances. Each small triangle represents the mean location of the first five sweeps of a single trial. The large circles show the locations of the landmarks, and the + shows the target location.

groups were apparent. Thus, there is no evidence that pigeons find one rule easier to learn than the other.

Overall, there appear to be several differences between the behavior of our pigeons and the behavior of the nutcrackers of Kamil and Jones (2000). The search behavior of nutcrackers using geometric rules appears to be more accurate than that of pigeons. Similar differences were obtained by Jones et al. (2002), with pigeons and nutcrackers learning to find the midway point between two landmarks. It is interesting to note that Olson (1991) found that the performance of nutcrackers in operant spatial nonmatching-to-sample tasks was also much better than that of pi- geons, suggesting there may be a general difference in the precision or accuracy of spatial information processing between these two species.

Second, pigeons in both our constant-bearing and constantdistance groups showed good transfer to novel interpolated interlandmark distances but not to novel extrapolated interlandmark distances. By contrast, nutcrackers trained in the constant-bearing condition showed excellent transfer to both the interpolated and extrapolated novel interlandmark distances (Kamil \& Jones, 2000). Nutcrackers in the constant-distance condition showed good transfer to interpolated distances but an increase in error for the extrap- 
olated distances. Thus, the pigeons in both groups responded similarly to nutcrackers trained with constant distances. Biegler, McGregor, and Healy (1998) suggested that animals could solve a geometric rule-learning task by learning a set of independent problems for each interlandmark distance. Their model allowed for accurate transfer to novel interpolated distances but not to novel extrapolated distances. Thus, it remains possible that nutcrackers in the constant-distance group and pigeons in both groups solved the task by learning separate problems rather than by learning a single rule.

There were also two differences in the details of the performance of the pigeons in this study and the nutcrackers in Kamil and Jones's (2000) study. First, the pigeons in the current study found the two problems, constant distance and constant direction, equally difficult. In contrast, nutcrackers found the constantbearing problem considerably easier than the constant-distance problem. Second, search accuracy was lower in the north-south axis (parallel to the landmark array) than in the east-west axis (perpendicular to the landmark array) for both groups of pigeons. It appears that pigeons had more difficulty localizing the midpoint between the landmarks than determining how far from the landmark array to search. In contrast, Kamil and Jones (2000) found no significant differences between axes in either the constant-bearing or the constant-distance group of nutcrackers.

These differences between pigeons and nutcrackers could reflect differences in apparatus and procedure. For example, in Kamil and Jones's (2000) study, the entrance to the search space was located on the east wall, which meant the nutcrackers initially approached the search space from the axis perpendicular to the array. In contrast, the start box in the present study was located on the north wall, which meant that the pigeons initially approached the search space from the axis parallel to the array. Additional research is needed to determine the effects of approach direction on the axis effect in each species.

These differences could also represent differences in how the species process spatial information when confronted with these geometric problems. For example, a variety of results with nutcrackers suggest that they are more likely to use information about bearings than information about distance under several different circumstances (Kamil \& Cheng, 2001; Kamil \& Jones, 1997, 2000), but there is no indication of such an effect in pigeons in either these data or in those of Jones et al. (2002). There are many studies in which results suggest species differences in spatial cognition between seed-storing and nonstoring birds, especially in tests of spatial memory (Brodbeck \& Shettleworth, 1995; Clayton \& Krebs, 1994; Kamil, Balda, \& Olson, 1994; Olson, Kamil, Balda, \& Nims, 1995). This ecological factor also deserves further investigation in the context of spatial orientation and navigation

In summary, our pigeons were able to solve search tasks involving complex constant-distance and constant-direction rules, but they were less accurate than nutcrackers and showed less complete transfer to novel interlandmark distances. Direct comparisons are compromised by slight differences in procedure and stimuli, but the lower accuracy for pigeons than reported previously for nutcrackers and the apparent species differences in the details of performance are consistent with the literature indicating a difference between pigeons and nutcrackers in spatial-information processing. Although pigeons' spatial search is sufficiently flexible that they can master a task that requires them to vary their distance or direction from landmarks across trials, this appears to be neither a preferred (Spetch et al., 1997) nor an easy strategy for pigeons.

\section{References}

Bennett, A. T. D. (1993). Spatial memory in a food storing corvid: I. Near tall landmarks are primarily used. Journal of Comparative Physiology, 173, 193-207.

Biegler, R., McGregor, D., \& Healy, S. D. (1998). How do animals "do" geometry? Animal Behaviour, 57, F4-F8

Brodbeck, D. R., \& Shettleworth, S. J. (1995). Matching location and color of a compound stimulus: Comparison of a food-storing and a nonstoring bird species. Journal of Experimental Psychology: Animal Behavior Processes, 21, 64-77.

Cheng, K. (1989). The vector sum model of pigeon landmark use. Journal of Experimental Psychology: Animal Behavior Processes, 15, 366-375.

Cheng, K. (1994). The determination of direction in landmark-based spatial search in pigeons: A further test of the vector sum model. Animal Learning \& Behavior, 22, 291-301.

Cheng, K. (1995). Landmark-based spatial memory in the pigeon. In D. L. Medin (Ed.), The psychology of learning and motivation (Vol. 33, pp. 1-21). San Diego, CA: Academic Press.

Cheng, K., \& Spetch, M. L. (1998). Mechanisms of landmark use in mammals and birds. In S. Healy (Ed.), Spatial representation in animals (pp. 1-17). Oxford, England: Oxford University Press.

Clayton, N. S., \& Krebs, J. R. (1994). One-trial associative memory: Comparison of food-storing and nonstoring species of birds. Animal Learning \& Behavior, 22, 366-372.

Collett, T. S., Cartwright, B. A., \& Smith, B. A. (1986). Landmark learning and visuo-spatial memories in gerbils. Journal of Comparative Physiology A, 158, 835-851.

Healy, S. (Ed.). (1998). Spatial representation in animals. Oxford, England: Oxford University Press.

Jones, J. E., Anotoniadis, E., Shettleworth, S. J., \& Kamil, A. C. (2002). A comparative study of geometric rule learning by nutcrackers (Nucifraga columbiana), pigeons (Columba livia), and jackdaws (Corvus monedula). Journal of Comparative Psychology, 116, 350-356.

Kamil, A. C., Balda, R. P., \& Olson, D. J. (1994). Performance of four seed-caching corvid species in the radial-arm maze analog. Journal of Comparative Psychology, 108, 385-393.

Kamil, A. C., \& Cheng, K. (2001). Way-finding and landmarks: The multiple-bearings hypothesis. Journal of Experimental Biology, 204, 103-113.

Kamil, A. C., \& Jones, J. E. (1997, November 20). The seed storing corvid Clark's nutcracker learns geometric relationships among landmarks. Nature, 390, 276-279.

Kamil, A. C., \& Jones, J. E. (2000). Geometric rule learning by Clark's nutcrackers (Nucifraga columbiana). Journal of Experimental Psychology: Animal Behavior Processes, 26, 439-453.

Keeton, W. T. (1974). The orientational and navigational basis of homing in birds. Advances in the Study of Behavior, 5, 47-132.

Kelly, D. M., Spetch, M. L., \& Heth, C. D. (1998). Pigeons' (Columba livia) encoding of geometric and featural properties of a spatial environment. Journal of Comparative Psychology, 112, 259-269.

Olson, D. J. (1991). Species differences in spatial memory among Clark's nutcrackers, scrub jays, and pigeons. Journal of Experimental Psychology: Animal Behavior Processes, 17, 363-376.

Olson, D. J., Kamil, A. C., Balda, R. P., \& Nims, P. J. (1995). Performance of four seed-caching corvid species in operant tests of nonspatial and spatial memory. Journal of Comparative Psychology, 109, 173-181.

Spetch, M. L., Cheng, K., \& MacDonald, S. E. (1996). Learning the configuration of a landmark array: I. Touch-screen studies with pigeons and humans. Journal of Comparative Psychology, 110, 55-68.

Spetch, M. L., Cheng, K., MacDonald, S. E., Linkenhoker, B. A., Kelly, 
D. M., \& Doerkson, S. R. (1997). Use of landmark configuration in pigeons and humans: II. Generality across search tasks. Journal of Comparative Psychology, 111, 14-24.

Spetch, M. L., \& Edwards, C. A. (1988). Pigeons', Columba livia, use of global and local cues for spatial memory. Animal Behaviour, 36, 293296

Spetch, M. L., \& Wilkie, D. M. (1994). Pigeons' use of landmarks presented in digitized images. Learning and Motivation, 25, 245-275.

Sutton, J. E., Olthof, A., \& Roberts, W. A. (2000). Landmark use by squirrel monkeys (Saimiri sciureus). Animal Learning \& Behavior, 28, $28-42$.
Wright, A. A., Cook, R. G., Rivera, J. J., Sands, S. F., \& Delius, J. D. (1988). Concept learning by pigeons: Matching-to-sample with trialsunique video picture stimuli. Animal Learning \& Behavior, 16, 436444.
Received January 2, 2002

Revision received May 1, 2002

Accepted May 2, 2002

\section{American Psychological Association} SUBSCRIPTION Claims INFORMATION

Today's Date:

We provide this form to assist members, institutions, and nonmember individuals with any subscription problems. With the appropriate information we can begin a resolution. If you use the services of an agent, please do NOT duplicate claims through them and directly to us. PLEASE PRINT CLEARLY AND IN INK IF POSSIBLE.

\begin{tabular}{llll}
\hline PRINT FULL NAME OR KEY NAME OF INSTTTUTION & & & \\
\hline ADDRESS & & & \\
\hline CITY & STATE/COUNTRY & \\
\hline
\end{tabular}

YOUR NAME AND PHONE NUMBER

TITLE

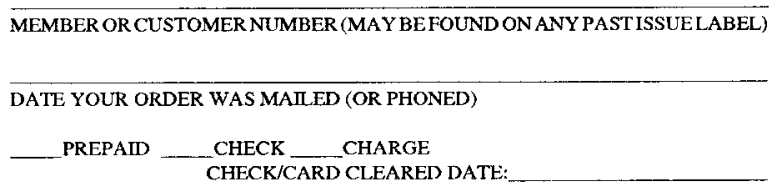

(If possible, send a copy, front and back, of your cancelled check to help us in our research of your claim.)

ISSUES: DAMAGED

\section{NUMBER OR MONTH}

Thank you. Once a claim is received and resolved, delivery of replacement issues routinely takes 4-6 weeks.

ACTION TAKEN:

STAFF NAME:
(TO BE FILLED OUT BY APA STAFF)

DATE OF ACTION:

INV. NO. \& DATE:

LABEL NO. \& DATE:

Send this form to APA Subscription Claims, 750 First Street, NE, Washington, DC 20002-4242

PLEASE DO NOT REMOVE. A PHOTOCOPY MAY BE USED. 